\title{
The effect of mono-diglycerides on the mechanical properties, microstructure and physical stability of an edible rice bran wax - gelatin biphasic gel system
}

\author{
Ariana Saffold ${ }^{1}$ and Nuria Acevedo ${ }^{2}$ \\ ${ }^{1}$ Iowa State University of Science and Technology \\ ${ }^{2}$ Iowa State Univeristy
}

February 18, 2022

\begin{abstract}
Bigels, are prepared by homogenization of an organogel and hydrogel. Bigels can offer improved properties when compared to other gelled systems due to its semi-solid internal phase and supramolecular interactions between components. The objective of this study was to determine the effect of mono-diglycerides (MDG) on the supramolecular structure and interactions, physical stability and mechanical properties of a rice bran wax (RBW)-gelatin bigels. The organogel-to-hydrogel (OG:HG) ratios tested were 60:40, 70:30 and 80:20 with MDG incorporated at four concentrations $(0.5,1,2,3 \%(\mathrm{w} / \mathrm{w}))$. Bigels were analyzed using nuclear magnetic resonance spectroscopy (NMR), confocal scanning laser microscopy (CSLM), Fourier transform infrared spectroscopy (FTIR), texture analysis and liquid binding capacity studies. The results showed that MDG have a dual effect on the structural organization and stability of the systems; they act as emulsifiers and/or crystallization modifiers. CSLM and FTIR showed that the addition of MDG had the greatest effect on the microstructure of the 60:40 OG:HG ratio, where MDG concentrations 0.5 and $1 \%(\mathrm{w} / \mathrm{w})$ exhibited a reduction in the size of the oleogel regions while concentrations 2 and $3 \%$ (w/w) showed a phase inversion. MDG addition resulted in a gradual decrease in bigel hardness, but an enhancement of physical stability.
\end{abstract}

\section{Introduction}

Bigels are biphasic gels systems in which both phases are structured semi-solids. Bigels contain both an aqueous hydrogel phase and a lipid organogel phase. Interest is growing in these biphasic gel systems because of their ability to address the limitations and issues associated with hydrogels, organogels and emulgels (Shakeel et al., 2018). Some of the advantages attributed to bigels include their ability to encapsulate both lipophilic and hydrophilic bioactive compounds (Behera et al., 2015); the capability to change both the aqueous and lipid phases to improve gel properties; and their enhanced physical stability due to a structured internal phase (Sahoo et al., 2015). Currently, bigels are mostly being considered for controlled delivery in pharmaceutical application, nonetheless, there is effort in investigating edible bigel formulations for food application. Specifically, there have been bigel formulations developed for encapsulation of probiotics to be incorporated into dairy products (Bollom et al., 2021; Zhuang et al., 2021). Other unique formulations have been developed to encapsulate sensitive bioactive reagents for application in functional food products (Zhu et al., 2021).

Even though bigels can be prepared without the use of surfactants or emulsifiers due to the semisolid nature of both phases, they may be susceptible to destabilization at higher temperatures. Destabilization at higher temperatures can occur when the sol-gel temperature is reached. At this temperature, both phases gain mobility and, similar to traditional emulsions, the mixture is thermodynamically unfavorable (Singh et al., 2014). In this case, there is most likely phase separation. To further enhance the stability of the biphasic gel 
systems, emulsifiers can be considered. The choice of emulsifier is important to promote successful stability of an emulsion and multi-phase systems with incompatible phases. There is a wide variety of emulsifiers available and their nature cannot only determine the stability of the system, but also impact emulsion formation and functional properties.

In the food industry, mono-diglycerides (MDG) and their derivatives are some of the most important food emulsifiers as they account for the majority $(\sim 70 \%)$ of emulsifier production. They are applied in foods such as bread, cake, spreads, ice cream and more. They exhibit more lipophilic behavior with an HLB value between 3-6 and are therefore more effective in stabilizing water-in-oil (W/O) emulsions (Norn et al., 2015). As MDG exhibit more lipophilic behavior, they are known to interact with the lipid phase. In addition, solid emulsifiers can affect nucleation and/or growth of fat crystals, accelerating or retarding these processes (Ribeiro et al., 2015). Monoglycerides, in particular, have been extensively studied as organogelators, as they are known to self-assemble into an inverse lamellar phase with close-packing when heated above its melting temperature and cooled again in oil. From these studies, it has been shown that the crystal morphology and role of monoglycerides in a system is dependent on the concentration, the type of oil it is added to and the presence of other components such as co-emulsifiers and/or co-gelators (Valoppi et al., 2017; Wang et al., 2022; Rodriguez-Hernandez et al., 2021).

Although there has been much work done on the effect of monoglycerides in organogel/oleogel and emulsiongel systems, there are few publications that have focused on their effect on bigel systems. The addition of MDG to a bigel may assist in extending physical stability, as well as providing another way to tailor and control the mechanical properties of the system. The goal of this study was to explore how MDG, a common food emulsifier, alters the interfacial interactions between the aqueous and lipid components of an edible rice bran wax (RBW) - gelatin bigel system. More specifically, in this study, it will be determined how different concentrations of MDG affect the RBW - gelatin bigel's microstructure, physical stability and mechanical properties at various oleogel-to-hydrogel (OG:HG) ratios.

\section{Experimental Procedures}

\subsection{Materials}

Pork skin gelatin, 250 bloom was donated by Nitta Gelatin, NA Inc. (Morrisville, NC). Rice bran wax was generously provided by Koster Keunen Inc. (Watertown, CT). Soybean oil was generously provided by ADM (Des Moines, IA). Pure glycerin flakes ( $42 \%$ monoglycerides, $58 \%$ diglycerides) were purchased from Modernist Pantry LLC.

\subsection{Sample Preparation}

\subsubsection{Oleogel Preparation}

For oleogel preparation, $10 \%(\mathrm{w} / \mathrm{w})$ RBW was combined with $90 \%(\mathrm{w} / \mathrm{w})$ soybean oil and heated to $90-95{ }^{\circ} \mathrm{C}$ with constant agitation. When all of the RBW melted, the mixture remained in the oven for an additional 15 minutes with constant stirring to erase crystal memory. In samples with MDG, MDG was added with RBW at the beginning of preparation and allowed to melt and dissolve in soybean oil before homogenization.

\subsubsection{Hydrogel Preparation}

A $7 \%(\mathrm{w} / \mathrm{w})$ gelatin concentration was used for this experiment. Gelatin was prepared by combining gelatin with deionized water at approximately $50^{\circ} \mathrm{C}$ for 30 minutes. The mixture was constantly stirred using a magnetic stirrer until complete dissolution.

\subsubsection{Bigel Preparation}

Bigels were prepared according to the method developed previously with some modifications (Saffold \& Acevedo, 2021). Briefly, three oleogel:hydrogel (OG:HG) ratios (60:40, 70:30, 80:20) with four concentrations of MDG $(0.5,1,2$ and $3 \%(\mathrm{w} / \mathrm{w}))$ were prepared. For bigel preparation, the gelatin mixture was brought to $65-70^{\circ} \mathrm{C}$ and the two phases were combined while at elevated temperatures. The mixture was immediately 
homogenized at 24,000 rpm for one minute and then placed in refrigerator $\left(4{ }^{\circ} \mathrm{C}\right)$ for 24 hours to allow gelation. Each sample was analyzed within five days of preparation. Triplicates were prepared and analyzed for each formulated bigel. Bigels without the addition of emulsifier were analyzed as controls. For shorthand, each formulation will be represented by their OG:HG ratio with the emulsifier concentration in parenthesis. For example, a 60:40 OG:HG bigel with an emulsifier concentration of $0.5 \%(\mathrm{w} / \mathrm{w})$ MDG will be referred to as "60:40(0.5)" and the OG:HG ratio 60:40 without emulsifier will be referred to as "60:40(0)". The oleogel and hydrogel concentrations remained at $10 \%(\mathrm{w} / \mathrm{w}) \mathrm{RBW}$ and $7 \%(\mathrm{w} / \mathrm{w})$ gelatin, respectively, for all bigel formulations.

\subsection{Analysis methods}

\subsubsection{Confocal Scanning Laser Microscopy (CSLM)}

Confocal microscopy was performed using Fluorescein isothiocyanate (FITC) and Nile Red to dye the gelatin HG and RBW-OG, respectively. FITC and Nile Red were added to each phase prior to gelation at 0.2 and 0.15 $\mathrm{g} / \mathrm{kg}$, respectively. Each bigel sample was cut into disks and kept cool before being placed on a glass bottom slide. Micrographs were acquired on an SP5 X MP Confocal Microscope (Leica Microsystems Inc.; Buffalo Grove, IL, USA) using a 40x objective magnification with no zoom. Excitation and emission wavelengths for FITC were 495 and 505-540 nm, respectively, and 552 and 565-700 nm for Nile Red, respectively. Images were 1024 x 1024 pixels and acquired at $200 \mathrm{~Hz}$. Signals were overlaid in Leica LAS AF Lite software (Leica Microsystems Inc.; Buffalo Grove, IL, USA). A sample with no dye was viewed as well (results not shown) to confirm that no autofluorescence was occurring from the bigel's natural constituents. A total of 5-7 images were obtained of each replicate.

\subsubsection{Fourier Transform Infrared Spectroscopy (FTIR)}

Spectra to analyze bigel microstructure were obtained using a Cary 630 FTIR spectrometer with a diamond ATR attachment (Agilent Technologies, Santa Clara, CA). A piece of the bigel was scooped out of the container and placed directly onto the stage, ensuring complete coverage of the spectrometer lens. The instrument scanned from $650-4000 \mathrm{~cm}^{-1}$ with a resolution of 74 scans at $4 \mathrm{~cm}^{-1}$. Two replicates were obtained for each formulation as results were nearly identical between replicates.

\subsubsection{Nuclear Magnetic Resonance Spectroscopy (NMR)}

All tests were performed with a flat-bottom glass NMR tube $(10 \mathrm{~mm}$ diameter, $180 \mathrm{~mm}$ length) and each formulation was tested in triplicate. Each tube was filled with bigel sample to a height of 2-in and were covered with parafilm to prevent moisture loss. Proton relaxation measurements were performed on a Bruker minispec mq-20 low-resolution spectrometer (Bruker, Biospin Corporation, Billerica, MA, USA) at a resonance frequency of $20 \mathrm{MHz}$. Before measurement, the samples were held at 22 in a high precision dry bath system for $30 \mathrm{~min}$. The NMR instrument was connected to a water bath circulator to keep the probe head at a constant temperature of 22 and ensure temperature did not contribute to differences in proton relaxation.

The Carr-Purcell-Meiboom-Gill (CPMG) pulse sequence was used to measure T2 between 0.2 and 2000 ms. Separation between the 90deg and 180deg pulses was 2.28 and $4.98 \mathrm{ls}$, respectively, and 500 data points were collected. For CPMG measurements, 8 scans were made with a 15.0-s recycle delay. Gain was adjusted for each sample to ensure the highest signal was close to $80 \%$. Relaxation curves were fitted to a continuous distribution of exponentials using the Inverse Laplace Transformation (ILT) algorithm, also referred as the CONTIN algorithm of Provencher (1982). Solid fat content (SFC) was also measured in all samples.

\subsubsection{Texture Analysis}

Prepared bigel samples were cut into cylinders that were $20-\mathrm{mm}$ in diameter and 10-mm in length. Before the test, the samples were allowed to sit at room temperature for 20 min in a capped container to prevent moisture loss. A texture profile analysis (TPA) was performed using a TA.XT plus texture analyzer (Stable Micro Systems, United Kingdom) equipped with a TA-25 attachment. The parameters of the TPA consisted of a $1 \mathrm{~mm} / \mathrm{s}$ test speed, $1 \mathrm{~mm} / \mathrm{s}$ post-test speed and a 3 -s holding period between the first and second cycle. 
The probe compressed the sample 5-mm in the longitudinal direction in each cycle. Samples were run in triplicate. The average hardness, springiness and cohesiveness were reported for each bigel formulation.

\subsubsection{Leaching Study}

Bigels were cut into disks (diameter: 20-mm; height: 3.2-mm) and weighed. The disk was then placed in-between a folded pre-weighed filter paper. A 200-g weight was placed on top of the disk for 30 min. At $30 \mathrm{~min}$, the disk was weighed again to determine the total amount of liquid lost from the sample. The filter paper was allowed to sit out for 24 hours to allow for the evaporation of any leached water. The filter paper was then weighed again to determine the amount of soybean oil leached from the sample. The total amount of liquid lost from the disk was calculated using the following formula:

$$
\% \text { Liquid Lost }=\frac{\text { Initial disk weight }- \text { Final disk weight }}{\text { Initial disk weight }} \times 100
$$

\subsubsection{Statistical Analysis}

A one-way ANOVA using Tukey's multiple comparisons was performed using RStudio software, $\mathrm{R}$ version 4.0.2 (RStudio; Boston, MA, USA). A statistically significant different was determined to have a p-value less than 0.05 .

\section{Results and Discussion}

\subsection{Bigel Appearance}

The successfully prepared bigel samples were evaluated based on their appearance after gelation. Bigels were classified as successful if they were opaque, white and showed no signs of phase separation. Fig. 1 shows the 60:40 and 70:30 OG:HG bigel formulations cut into a cylinder shape. 80:20 OG:HG formulations (not shown) could not be stabilized and showed phased separation immediately after preparation despite the addition of MDG. For this reason, 80:20 OG:HG were excluded from further testing. Most of the formulations for 60:40 and 70:30 OG:HG were successful with the exception of 70:30(3) as can be observed in Fig. 1. The 70:30(3) bigel formulation resulted in phase separation, whereas the control, 70:30(0), showed excellent physical stability. With an increase in MDG concentration, going from $0 \%$ to $3 \%$, the bigel became more malleable for both 60:40 and 70:30 OG:HG ratios. The presence of MDG creates a softer, more pliable bigel without altering the visual appearance from the control and without the use of a large amount of hydrogel which can be beneficial for applications where more lipidic phase is desirable. The fact that MDG creates a softer, more malleable bigel may be attributed to MDG being a known lipid modifier (Ribeiro et al., 2015; Alfutimie et al., 2016). It can be hypothesized that MDG may have a dual effect on the bigel system, serving as both a lipid modifier in the lipid phase and an emulsifier at the aqueous-lipid interface.

\subsection{Confocal Scanning Laser Microscopy (CSLM)}

The bigels' microstructure for 60:40 and 70:30 OG:HG ratios is shown in Fig. 2. The lipid and aqueous phases are depicted in red and green, respectively. The images shown were carefully chosen as representatives of what was observed across all the images taken for each sample. The 60:40(0) sample shows large oleogel regions dispersed in a hydrogel matrix, indicating this sample is an oleogel-in-hydrogel type bigel. In the literature, an oleogel-in-hydrogel type of bigel describes oleogel droplets dispersed in a hydrogel matrix, but in this formulation, the term "droplets" is not an accurate descriptor for the oleogel phase because the oleogel portion is quite large and irregularly shaped. When $0.5 \%(\mathrm{w} / \mathrm{w}) \mathrm{MDG}$ is added to the 60:40 OG:HG formulation, there is a clear reduction in the size of the oleogel regions. This reduction in oleogel region size is evidence that MDG is working as an emulsifier at the lipid-aqueous interface. At $1 \%$ and $2 \%(\mathrm{w} / \mathrm{w}$ ) MDG, the bigel transitions from an oleogel-in-hydrogel type (1\% (w/w) MDG) to a bi-continuous bigel type $(2 \%(\mathrm{w} / \mathrm{w}) \mathrm{MDG})$ in which neither phase forms droplets, but rather both phases create a bi-continuous matrix. The 60:40(3) bigel formulation shows a phase inversion where the hydrogel is now dispersed in an oleogel matrix. On the other hand, all 70:30 OG:HG formulations, regardless of the amount of MDG added, 
appear to be bi-continuous bigels where there is no clear distinction as to which phase is the continuous or dispersed phase (Lupi et al., 2016; Shakeel et al., 2018). A bi-continuous gel can also be referred to as a matrix-in-matrix type of bigel. As mentioned previously, the 70:30(3) formulation could not be stabilized and showed clear phase separation.

In addition to the microstructural changes, MDG also had an unexpected effect most evident in formulations with high MDG concentrations. Fig. 2 shows the formation of spherical structures that appeared in the lipid phase with sizes ranging from $10-\mu \mathrm{m}$ to $40-\mu \mathrm{m}$. These spherical structures are evident in small amounts in 70:30(0), 70:30(0.5) and 70:30(1) bigels; nevertheless, they become very noticeable in 60:40(3) and 70:30(2) bigels. Observation under polarized light microscopy (PLM; not shown) confirmed that these spherical structures were not spherulites resulting from the crystallization of MDG. Therefore, it can be hypothesized that they are lipid droplets that were not incorporated into an oleogel region or matrix. Whether or not these droplets contain rice bran wax is still unclear, but PLM did not show rice bran wax crystals within these droplets. Additional studies are necessary to confirm the contents of these lipid droplets and why they form in greater numbers when MDG is present at high concentrations.

\subsection{Fourier Transform Infrared Spectroscopy (FTIR)}

FTIR was used to investigate potential interactions among components of all phases; the oleogel, hydrogel and MDG. Fig. 3 shows the spectra for the 60:40 and 70:30 OG:HG formulations. The broad peak at $\sim 3370$ $\mathrm{cm}^{-1}$ is associated with $\mathrm{O}-\mathrm{H}, \mathrm{N}-\mathrm{H}$ stretching and hydrogen bonding. This peak can mostly be attributed to water molecules (Sagiri et al., 2015) and hydroxyl groups on gelatin amino acid residues and MDG (Mohammed et al., 2018). Peaks at ${ }^{\sim} 2920 \mathrm{~cm}^{-1}$ and $\sim 2850 \mathrm{~cm}^{-1}$ are associated with $\mathrm{C}-\mathrm{H}$ stretching in RBW, soybean oil and MDG (Mohammed et al., 2018; Wakhet et al., 2015; Ishaka et al., 2014). Peaks ${ }^{\sim} 2100 \mathrm{~cm}^{-1}$ and $\sim 1635 \mathrm{~cm}^{-1}$ are associated with amino acid groups in gelatin and were confirmed by a spectrum of pure $7 \%\left(\mathrm{w} / \mathrm{w}\right.$ ) gelatin (not shown). Peaks ${ }^{\sim} 1740 \mathrm{~cm}^{-1}$ and in the region of below $1600 \mathrm{~cm}^{-1}$ are associated with carbonyl groups stretching and C-H bending, respectively, in RBW, soybean oil and MDG (Mohammed et al., 2018; Wakhet et al., 2015; Ishaka et al., 2014).

There were noticeable changes in peak intensity which can be attributed to the presence of MDG in the samples. In general, in both 60:40 and 70:30 OG:HG ratios, the peaks attributed to MDG and the oleogel phase increased in intensity as the MDG concentration increased. Simultaneously, peaks associated with the aqueous phase decreased in intensity with increasing MDG concentration. From these observations, it appears that interactions within the aqueous phase are decreasing in strength while interactions within the lipid phase are increasing in strength with the addition of MDG. For 60:40 OG:HG formulations, there was a gradual decline in peak intensity at $3368 \mathrm{~cm}^{-1}$ which may indicate a decrease in hydrogen bonding or diminishing strength in hydrogen bonds (Yilmaz \& Ogutcu, 2014). This is an unexpected result because monoglycerides, when crystallized, have an inverse bilayer structure stabilized by hydrogen bonds established by its secondary and primary -OH groups. (Aguilar-Zarate et al., 2019). It would be expected that the peak at $3368 \mathrm{~cm}^{-1}$ would increase in intensity due to the presence of additional -OH groups from MDG or the establishment of new hydrogen bonds from MDG crystallization. This decrease in this peak's intensity may indicate that MDG weakened or reduced the density of hydrogen bonds in the hydrogel matrix, eventually leading to a bigel phase inversion shown in the 60:40(3) formulation in Fig. 2. MDG crystallization could still be happening at the same time, but the hydrogen bonding associated with this process may be lesser in comparison to the dissociation of the hydrogel's hydrogen bonding, leading to a net decrease in the peak intensity. The same phenomenon is also seen for the 70:30 OG:HG formulations, but the decrease in hydrogel associated peak intensities is most prominent in the 70:30(2) formulation. Unlike the 60:40 OG:HG ratio, the decrease in hydrogel peak intensities did not lead to a phase inversion in 70:30 OG:HG, but it is notable that 70:30(3) could not be stabilized for reasons that are not yet clear.

In contrast, an increase in peak intensities associated with the oleogel phase was observed for both 60:40 and 70:30 OG:HG ratios, with more prominent changes noticeable in 60:40 OG:HG formulations. This increase in intensity may just indicate that MDG is acting as an organogelator, strongly associating with the oleogel phase and/or strengthening the interactions of the oleogel matrix. This would agree with the formation of a 
continuous matrix in the 60:40(3) formulation seen in Fig. 2. The exception to this pattern is the 60:40(0.5) formulation where, not only is there a decrease in oleogel associated peaks, but there is a decrease in oleogel region size in Fig. 2. When the regions are smaller, there is less interaction within the oleogel phase because a larger area of the lipid interface is surrounded by the continuous hydrogel matrix. The data from Figs. 2 and 3 suggest that the emulsification capacity for MDG in this bigel system is greatest at $0.5 \%(\mathrm{w} / \mathrm{w})$ MDG and a OG:HG ratio of 60:40. Beyond 0.5\% (w/w), MDG appears to have greater interactions in the oleogel phase, as an organogelator, than at the lipid-aqueous interface as an emulsifier.

\subsection{Nuclear Magnetic Resonance Spectroscopy (NMR)}

\subsubsection{Solid fat content $(S F C)$}

SFC was measured to determine if MDG was contributing to the solid content of the bigel and played a role in promoting crystallization of the lipid phase. Fig. 4 shows normalized SFC for all bigel formulations. Normalization was achieved by measuring the SFC of a pure $7 \%(\mathrm{w} / \mathrm{w})$ gelatin sample and subtracting its value from all bigel SFC measurements to ensure that the data only reflects the SFC of the sample. When comparing the expected theoretical SFC of the samples, accounting for the addition of MDG solids, with the experimental data obtained, it was found that the actual SFC values were lower than expected. For example, for the formulation 70:30(0) and 70:30(1), the expected SFC values were calculated to be $7.0 \%$ and $7.9 \%$. The measured values for these samples were $6.0 \% \pm 0.4 \%$ and $6.2 \% \pm 0.2 \%$, respectively. These results can be explained by the solubility of RBW in liquid oil, determined to be $93.05 \%$, and the presence of resins which can affect the solid-fat content (Blake et al., 2014). The results of this study are in line with those obtained by Blake et al. (2014) for RBW.

In contrast, although the experimental SFC values were lower than theoretically expected, an additional increase in the experimental SFC values was observed with an increase of MDG concentration. For 60:40 OG:HG, there appears to be a break in the positive linear trend with an unexpected increase in SFC at $2 \%$ and $3 \%(\mathrm{w} / \mathrm{w})$ MDG. There could be two plausible explanations for this. One explanation is that MDG at $2 \%(\mathrm{w} / \mathrm{w})$ and higher concentrations is could be promoting crystallization of RBW. Emulsifiers can be used to modify crystallization properties of fats. They can act as heteronuclei and accelerate fat crystal growth through a catalytic action as impurities (Ribeiro et al., 2015). The SFC data provides evidence that this could be the case with RBW as well. Another explanation is that MDG could be co-crystallizing at these concentrations. Previous studies have shown that monoglycerides can co-crystallize with wax-based oleogel systems, producing highly birefringent structures (Rodriguez-Hernandez et al., 2021; Toro-Vazquez et al., 2013). In both cases, crystal growth of RBW and MDG can be hindered. If MDG is accelerating RBW crystal growth, it is well known that accelerated crystallization creates smaller crystals (Ribeiro et al., 2015). In the case of MDG co-crystallization, MDG crystal growth can be restricted once they reach the interface of the hydrogel phase or the RBW crystal network (Rodriguez-Hernandez et al., 2021). In particular, RBW requires long crystals to promote crystal-crystal interactions that are considered desirable for the formation of strong organogels (Blake et al., 2014; Dassanayake et al. 2012). Knowing that long crystals are desirable for stronger RBW organogel matrices, it can be hypothesized that the addition of MDG will reduce the strength of the overall bigel system which may impact its stability. This is discussed further with the results obtained from texture analysis and the leaching study.

\subsubsection{Spin-spin Relaxation $\left(T_{2}\right)$}

$\mathrm{T}_{2}$ relaxation spectra were obtained to determine the effect of MDG on molecular mobility in the bigel matrices. $\mathrm{T}_{2}$ relaxation times reflect the mobility of proton populations within the matrix, where a shorter $\mathrm{T}_{2}$ indicates a less mobile population and a longer $\mathrm{T}_{2}$ indicates a more mobile population (Luyts et al., 2013). In 60:40 OG:HG bigels, regardless of the MDG proportion added, three populations with varying degrees of mobility were observed (Fig. 5). To confirm the identity of these populations, spectra of pure $10 \%$ $(\mathrm{w} / \mathrm{w})$ oleogel and $7 \%(\mathrm{w} / \mathrm{w})$ gelatin sample were obtained (results not shown). The least mobile proton population with a $\mathrm{T}_{2,1}$ of $\sim 44 \mathrm{~ms}$ is associated with the matrices of both the aqueous and lipid phases. These protons are most likely more tightly bound and strongly associated with the components of the oleogel and 
hydrogel phases. The second, more mobile population with a $\mathrm{T}_{2,2}$ of $\sim 180 \mathrm{~ms}$ is likely associated with the lipid phase. This may involve the oleogel and the MDG molecules. The third proton population, the most mobile, has a $T_{2,3}$ of $\sim 525-630 \mathrm{~ms}$ that can be attributed to protons of the aqueous phase, or hydrogel. This population shows to be affected the greatest by the addition of MDG. When comparing the most mobile proton population, $\mathrm{T}_{2,3}, 60: 40(0.5)$ does not show much difference compared to the control, 60:40(0); however there is a noticeable difference at higher MDG concentrations. The samples 60:40(1) and 60:40(2) continually decreased in overall molecular mobility which is depicted by the low values of $\mathrm{T}_{2,3}$. This decrease in mobility is most likely related to the increased SFC (Fig. 4) in the lipid phase. If the MDG are increasing the solids content and promoting crystallization of RBW, then the solids may be restricting the movement of the aqueous phase. Breaking from the trend of decreasing mobility in $\mathrm{T}_{2,3}, 60: 40(3)$ is less mobile than 60:40(0) but is more mobile than 60:40(1) and 60:40(2). The 60:40(3) sample may have this increase in mobility because of its change in microstructure. The 60:40(3) sample was found to have a different microstructure than the other 60:40 OG:HG formulations (Fig. 2) where it was a hydrogel-in-oleogel type of bigel rather than an oleogel-in-hydrogel type. It was also found by FTIR (Fig. 3) that 60:40(3) has a decrease in interactions within the hydrogel phase. These decreased interactions within the hydrogel phase and discontinuation of the hydrogel matrix, may lead the aqueous phase in 60:40(3) to have more mobility.

On the other hand, 70:30(0) was found to have four populations. The first three populations are hypothesized to have the same identities as in the 60:40 OG:HG formulations. The $\mathrm{T}_{2}$ values for the first three peaks in all the 70:30 OG:HG bigels are $\sim 45 \mathrm{~ms}, \sim 150 \mathrm{~ms}$ and $\sim 500 \mathrm{~ms}$. It should be noted that the $\mathrm{T}_{2,3}$ peaks in the 70:30 OG:HG formulations were less mobile than those in 60:40 OG:HG formulations. Similarly to what was observed fro the 60:40 OG:HG formulations, the addition of MDG influenced the $\mathrm{T}_{2,3}$ values although at much lesser extent. This may be due to a dilution effect or it may have a connection to the microstructure where 70:30 OG:HG formulations consistently exhibited a bi-continuous matrix, whereas there were greater microstructural changes in 60:40 OG:HG formulations (Fig. 2). The higher SFC content in the 70:30 OG:HG ratio compared to 60:40 OG:HG may also be contributing to restricted movement of the aqueous phase in the third population. The fourth population in 70:30(0) with a $\mathrm{T}_{2,4}$ of $\sim 1320 \mathrm{~ms}$ is most likely attributed to the aqueous phase because the pure gelatin sample was found to have a $T_{2}$ as high as $\sim 850 \mathrm{~ms}$ while the pure oleogel sample did not surpass a $\mathrm{T}_{2}$ of $\sim 230 \mathrm{~ms}$. The $\mathrm{T}_{2,4}$ value is greater than that of what was found in pure gelatin and the reason for its appearance is still unclear. Further investigation is needed to understand how this fourth peak is related to the microstructure of this formulation. Overall, MDG seem to have the most impact on the mobility of molecules associated with the aqueous phase.

\subsection{Texture Analysis}

Texture analysis was performed to determine how mechanical properties of the sample were affected with the addition of MDG. Table 1 shows values for hardness, springiness and cohesiveness for 60:40 and 70:30 OG:HG bigel formulations. Additional texture anlaysis parameters are shown in Table S1. Hardness is shown to significantly decrease with the addition of MDG for both OG:HG ratios with 3\% (w/w) MDG having the lowest hardness values. This is an unexpected finding since analysis of SFC (Fig. 4) showed increased SFC with the addition of MDG. With an increased SFC, it is expected that the hardness would increase due to more solids and structure in the sample. Instead, even with the addition of more solids, there is a clear and significant decrease in sample hardness. These results agree with previous studies, where the addition of high proportions of monoglycerides were found to decrease the hardness and/or mechanical strength of various gel systems (Rodriguez-Hernandez, 2021; Toro-Vazquez et al., 2013; Du et al., 2021). The amount of monoglycerides that were needed to cause this effect is dependent on the components of the system. Comparing the highest and lowest MDG concentrations for the 60:40 and 70:30 OG:HG ratios, the average hardness decreased by $68 \%$ and $31 \%$, respectively. This data provides evidence of MDG playing the role of lipid modifier and/or a co-crystallizer in the oleogel phase. RBW, when forming an oleogel, is known for its effective needle-like or fibrous crystals that interact and form a matrix to entrap large amounts of oil (Blake et al., 2014; Dassanayake et al., 2012). The crystal size grows proportionately with RBW concentration and correlates to an increased hardness (Dassanayake et al., 2012). The addition of MDG may be interrupting the interactions between RBW crystals or inhibiting crystal growth size, causing the hardness of the gel to 
decrease as discussed with SFC results (Fig. 4). MDG can also act as seeds for nucleation and accelerate the crystallization process, as mentioned before (Ribeiro et al., 2015). This process can also inhibit crystal growth size. This decrease in hardness may be related to a decreased in crystal-crystal interactions in RBW which are known to strengthen the RBW organogel matrix (Blake et al., 2014; Dassanayake et al., 2012).

\subsection{Leaching Study}

A leaching test was performed to assess the physical stability of the bigel formulations (Table 2). It was found that for all formulations, the amount of liquid lost remained below $10 \%$ with greater than $90 \%$ of leached liquid coming from the aqueous phase. In NMR T2-relaxation, the aqueous phase was found to have a more mobile population than the lipid phase. The observation that the aqueous phase accounts for most of the liquid leached from the system, is consistent with the fact that the aqueous phase is more mobile within the bigel and, therefore, water tends to percolate more than the oil from the bigel matrix. Data from the leaching study also found that formulations with the highest concentrations of MDG had a significantly lower amount of overall liquid leached from the matrix. Specifically, the amount of water that leached from the system reduced by $34 \%$ and $23 \%$ when comparing the highest and lowest MDG concentrations for OG:HG ratios 60:40 and 70:30, respectively. This may be the result of MDG interacting with the aqueous-lipid interface or the co-crystallization of MDG preventing the movement/leaching of water from the matrix. Additional studies are needed to confirm which mechanism is at work in the system. Although texture analysis showed a decrease in hardness, this does not necessarily correspond to a loss of physical stability.

\section{Conclusion}

The effect of mono-diglycerides (MDG) on a rice bran wax oleogel - gelatin hydrogel biphasic system was explored to improve its physical stability and tailor its mechanical and textural properties. MDG had the most significant impact on the 60:40 OG:HG bigel formulations, where CSLM images confirmed a phase inversion from oleogel-in-hydrogel to hydrogel-in-oleogel at 3\% (w/w) MDG. These microstructural changes were reflected in FTIR to show that as the MDG concentration increased, the interactions within the oleogel phase increased and the interactions within the hydrogel decreased. The textural hardness of the bigel formulations also significantly decreased with the increase of MDG concentration while maintaining greater physical stability. Through the available evidence, it appears MDG serves as both a lipid modifier and emulsifier in this biphasic system and can be used to both tailor the mechanical properties and increase physical stability.

\section{Acknowledgements}

This paper is a product of the Iowa Agriculture and Home Economics Experiment Station, Ames, Iowa. Project No. IOW03902 sponsored by Hatch Act and State of Iowa funds and USDA National Institute of Food and Agriculture NNF award \# 2017-38420-26769.

\section{Competing Interests}

The authors declare no competing interests.

\section{Data Availability}

All data generated or analyzed during this study are included in this published article and its supplementary information files.

\section{References}

Aguilar-Zárate, M., De la Peña-Gil, A., Álvarez-Mitre, F. M., Charó-Alonso, M. A., \& Toro- Vazquez, J. F. (2019). Vegetable and Mineral Oil Organogels Based on Monoglyceride and Lecithin Mixtures.Food Biophysics , 14 (3), 326-345. https://doi.org/10.1007/s11483-019-09583-1

Alfutimie, A., Al-Janabi, N., Curtis, R., \& Tiddy, G. J. T. (2016). The Effect of monoglycerides on the crystallisation of triglyceride. Colloids and Surfaces A: Physicochemical and Engineering Aspects ,494, 170179. https://doi.org/10.1016/j.colsurfa.2016.01.029 
Behera, B., Sagiri, S. S., Pal, K., Pramanik, K., Rana, U. A., Shakir, I., \& Anis, A. (2015). Sunflower oil and protein-based novel bigels as matrices for drug delivery applications - Characterization and in vitro antimicrobial efficiency. Polymer-Plastics Technology and Engineering , 54 (8), 837-850.

Blake, A. I., Co, E. D., \& Marangoni, A. G. (2014). Structure and Physical Properties of Plant Wax Crystal Networks and Their Relationship to Oil Binding Capacity. Journal of the American Oil Chemists' Society, 91 (6), 885-903. https://doi.org/10.1007/s11746-014-2435-0

Bollom, M. A., Clark, S., \& Acevedo, N. C. (2021). Edible lecithin, stearic acid, and whey protein bigels enhance survival of probiotics during in vitro digestion. Food Bioscience, 39 . https://doi.org/10.1016/j.fbio.2020.100813

Dassanayake, L. S. K., Kodali, D. R., Ueno, S., \& Sato, K. (2012). Crystallization Kinetics of Organogels Prepared by Rice Bran Wax and Vegetable Oils. Journal of Oleo Science , 61 (1), 1-9. https://doi.org/10.5650/jos.61.1

Ishaka, A., Umar Imam, M., Mahamud, R., Zuki, A. B. Z., \& Maznah, I. (2014). Characterization of rice bran wax policosanol and its nanoemulsion formulation. International Journal of Nanomedicine ,9 , 2261-2269. https://doi.org/10.2147/IJN.S56999

Lupi, F. R., Shakeel, A., Greco, V., Oliviero Rossi, C., Baldino, N., \& Gabriele, D. (2016). A rheological and microstructural characterisation of bigels for cosmetic and pharmaceutical uses. Materials Science and Engineering: C , 69 , 358-365. https://doi.org/10.1016/j.msec.2016.06.098

Luyts, A., Wilderjans, E., Waterschoot, J., Van Haesendonck, I., Brijs, K., Courtin, C. M., Hills, B., \& Delcour, J. A. (2013). Low resolution 1H NMR assignment of proton populations in pound cake and its polymeric ingredients. Food Chemistry , 139 (1), 120-128. https://doi.org/10.1016/j.foodchem.2013.01.062

Mohammed, I. A., Shahabuddin, S., Khanam, R., \& Saidur, R. (2018). Synthesis, characterization and antibacterial activity of novel poly(silyl ether)s based on palm and soy oils. Polímeros ,28 , 406-412. https://doi.org/10.1590/0104-1428.10317

Norn, V., Moonen, H., \& Bas, H. (2015). Mono- and Diglycerides. InEmulsifiers in food technology (2 ${ }^{\text {nd }}$ ed., pp. 73-91). Chichester, English:Wiley Blackwell.

Ribeiro, A. P. B., Masuchi, M. H., Miyasaki, E. K., Domingues, M. A. F., Stroppa, V. L. Z., de Oliveira, G. M., \& Kieckbusch, T. G. (2015). Crystallization modifiers in lipid systems. Journal of Food Science and Technology , 52 (7), 3925-3946. https://doi.org/10.1007/s13197-014-1587-0

Rodríguez-Hernández, A. K., Pérez-Martínez, J. D., Gallegos-Infante, J. A., Toro-Vazquez, J. F., \& OrnelasPaz, J. J. (2021). Rheological properties of ethyl cellulose-monoglyceride- candelilla wax oleogel vis-a-vis edible shortenings. Carbohydrate Polymers , 252 , 117171. https://doi.org/10.1016/j.carbpol.2020.117171

Saffold, A. C., Acevedo, N. C. (2021). Development of Novel Rice Bran Wax/Gelatin-Based Biphasic Edible Gels and Characterization of their Microstructural, Thermal, and Mechanical Properties. Food and Bioprocess Technology . https://doi.org/10.1007/s11947-021-02719-7

Sagiri, S. S., Singh, V. K., Kulanthaivel, S., Banerjee, I., Basak, P., Battachrya, M. K., \& Pal, K. (2015). Stearate organogel-gelatin hydrogel based bigels: Physicochemical, thermal, mechanical characterizations and in vitro drug delivery applications. Journal of the Mechanical Behavior of Biomedical Materials , 43 , 1-17. https://doi.org/10.1016/j.jmbbm.2014.11.026

Sahoo, S., Singh, V. K., Uvanesh, K., Biswal, D., Anis, A., Rana, U. A., Al-Zahrani, S. M., \& Pal, K. (2015). Development of ionic and non-ionic natural gum-based bigels: Prospects for drug delivery application. Journal of Applied Polymer Science, 132 (38). https://doi.org/10.1002/app.42561

Shakeel, A., Lupi, F. R., Gabriele, D., Baldino, N., \& De Cindio, B. (2018). Bigels: A unique class of materials for drug delivery applications. Soft Materials , 16 (2), 77-93. 
https://doi.org/10.1080/1539445X.2018.1424638

Singh, V. K., Banerjee, I., Agarwal, T., Pramanik, K., Bhattacharya, M. K., \& Pal, K. (2014). Guar gum and sesame oil based novel bigels for controlled drug delivery. Colloids and Surfaces B: Biointerfaces ,123, 582-592. https://doi.org/10.1016/j.colsurfb.2014.09.056

Toro-Vazquez, J. F., Mauricio-Perez, R., Gonzalez-Chavez, M. M., Sanchez-Becerril, M., Ornelas-Paz, J. de J., \& Perez-Martinez, J. D. (2013). Physical properties of organogels and water in oil emulsions structured by mixtures of candelilla wax and monoglycerides. Food Research International , 54 (2), 1360-1368. https://doi.org/10.1016/j.foodres.2013.09.046

Valoppi, F., Calligaris, S., Barba, L., Šegatin, N., Poklar Ulrih, N., \& Nicoli, M. C. (2017). Influence of oil type on formation, structure, thermal, and physical properties of monoglyceride-based organogel.European Journal of Lipid Science and Technology , 119 (2), 1500549. https://doi.org/10.1002/ejlt.201500549

Wakhet, S., Singh, V. K., Sahoo, S., Sagiri, S. S., Kulanthaivel, S., Bhattacharya, M. K., Kumar, N., Banerjee, I., \& Pal, K. (2015). Characterization of gelatin-agar based phase separated hydrogel, emulgel and bigel: A comparative study. Journal of Materials Science: Materials in Medicine , 26 (2), 118. https://doi.org/10.1007/s10856-015-5434-2

Wang, S., Chen, K., \& Liu, G. (2022). Monoglyceride oleogels for lipophilic bioactive delivery - Influence of self-assembled structures on stability and in vitro bioaccessibility of astaxanthin. Food Chemistry , 375 , 131880. https://doi.org/10.1016/j.foodchem.2021.131880

Yılmaz, E., \& Ögütcü, M. (2014). Properties and Stability of Hazelnut Oil Organogels with Beeswax and Monoglyceride. Journal of the American Oil Chemists' Society , 91 (6), 1007-1017. https://doi.org/10.1007/s11746-014-2434-1

Zhuang, X., Clark, S., \& Acevedo, N. (2021). Bigels - Oleocolloid matrices - As probiotic protective systems in yogurt. Journal of Food Science , 86 (11), 4892-4900. https://doi.org/10.1111/1750-3841.15928

Zhu, Z., Wen, Y., Yi, J., Cao, Y., Liu, F., \& McClements, D. J. (2019). Comparison of natural and synthetic surfactants at forming and stabilizing nanoemulsions: Tea saponin, Quillaja saponin, and Tween 80.Journal of Colloid and Interface Science , 536 , 80-87. https://doi.org/10.1016/j.jcis.2018.10.024

\section{Figure Legends}

Fig. 1 All 60:40 and 70:30 OG:HG bigel formulations cut into cylinders

Fig. 2 Images of the microstructure of 60:40 and 70:30 OG:HG bigels formulated with obtained by CSLM. The controls, lowest and highest successful concentrations of each ratio are shown. The red droplets represent the oleogel phase while the hydrogel phase appears green

Fig. 3 FTIR spectra of all successful bigel formulations

Fig. 4 Solid-fat content measurements obtained with low resolution NMR for all successful bigel formulations

Fig. 5 NMR $T_{2}$ relaxation spectra for all successful bigel formulations

Table 1. Hardness, springiness and cohesiveness measurements for all successful bigel formulations. Means with the same letter are not significantly different $(\mathrm{p}<0.05)$.

\begin{tabular}{llllll}
\hline & OG:HG Ratio & OG:HG Ratio & OG:HG Ratio & OG:HG Ratio & OG \\
\hline Emulsifier Concentration [\% (w/w)] & $\mathbf{6 0 : 4 0}$ & $\mathbf{7 0 : 3 0}$ & $\mathbf{6 0 : 4 0}$ & $\mathbf{7 0 : 3 0}$ & $\mathbf{6 0 : 4}$ \\
& Hardness (g) & Hardness (g) & Springiness & Springiness & Coh \\
0 & $2680 \pm 163^{\mathrm{a}}$ & $2444 \pm 357^{\mathrm{ab}}$ & $0.85 \pm 0.03^{\mathrm{a}}$ & $0.65 \pm 0.04^{\mathrm{a}}$ & 0.65 \\
0.5 & $2632 \pm 297^{\mathrm{a}}$ & $2558 \pm 327^{\mathrm{a}}$ & $0.70 \pm 0.05^{\mathrm{b}}$ & $0.66 \pm 0.02^{\mathrm{a}}$ & 0.61 \\
1 & $2064 \pm 119^{\mathrm{ab}}$ & $2200 \pm 18^{\mathrm{ab}}$ & $0.76 \pm 0.01^{\mathrm{b}}$ & $0.70 \pm 0.03^{\mathrm{ab}}$ & 0.62
\end{tabular}




\begin{tabular}{llllll}
\hline & OG:HG Ratio & OG:HG Ratio & OG:HG Ratio & OG:HG Ratio & OG \\
\hline 2 & $1515 \pm 86^{\mathrm{b}}$ & $1688 \pm 95^{\mathrm{b}}$ & $0.88 \pm 0.03^{\mathrm{a}}$ & $0.75 \pm 0.01^{\mathrm{b}}$ & 0.56 \\
3 & $849 \pm 54^{\mathrm{c}}$ & N/A & $0.95 \pm 0.01^{\mathrm{c}}$ & N/A & 0.62 \\
\hline
\end{tabular}

${ }^{*}$ HG: Hydrogel

*OG: Oleogel

Table 2. Percentages of total liquid lost (combination of water and oil), water lost and soybean oil lost in the sample after 30 min. Means with the same letter are not significantly different $(\mathrm{p}<0.05)$.

\begin{tabular}{lllll}
\hline & OG:HG Ratio & OG:HG Ratio & OG:HG Ratio & OG:HG Ratio \\
\hline Emulsifier Concentration [\% (w/w)] & $\mathbf{6 0 : 4 0}$ & $\mathbf{7 0 : 3 0}$ & $\mathbf{6 0 : 4 0}$ & $\mathbf{7 0 : 3 0}$ \\
& \% Liquid Lost & \% Liquid Lost & \% Water Lost & \% Water Lost \\
0 & $6.9 \pm 0.3^{\mathrm{a}}$ & $5.9 \pm 0.1^{\mathrm{a}}$ & $6.4 \pm 0.5^{\mathrm{a}}$ & $4.4 \pm 0.8^{\mathrm{a}}$ \\
0.5 & $6.4 \pm 0.3^{\mathrm{ab}}$ & $5.8 \pm 1.2^{\mathrm{a}}$ & $5.9 \pm 0.1^{\mathrm{a}}$ & $4.0 \pm 0.2^{\mathrm{a}}$ \\
1 & $5.5 \pm 0.2^{\mathrm{b}}$ & $4.9 \pm 0.2^{\mathrm{a}}$ & $5.1 \pm 0.7^{\mathrm{a}}$ & $3.8 \pm 0.6^{\mathrm{a}}$ \\
2 & $5.4 \pm 0.1^{\mathrm{b}}$ & $5.1 \pm 0.1^{\mathrm{a}}$ & $4.7 \pm 0.4^{\mathrm{a}}$ & $3.4 \pm 0.7^{\mathrm{a}}$ \\
3 & $5.8 \pm 0.2^{\mathrm{a}}$ & N/A & $4.2 \pm 1.0^{\mathrm{b}}$ & N/A \\
\hline
\end{tabular}

*HG: Hydrogel

*OG: Oleogel

\section{Hosted file}

Supplementary Table.docx available at https://authorea.com/users/461167/articles/556922-theeffect-of-mono-diglycerides-on-the-mechanical-properties-microstructure-and-physicalstability-of-an-edible-rice-bran-wax-gelatin-biphasic-gel-system

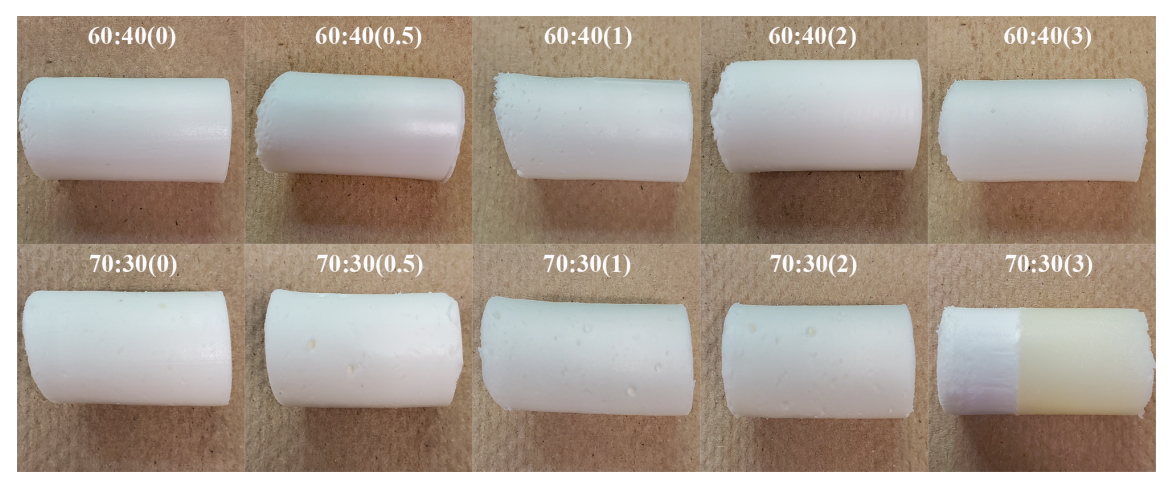



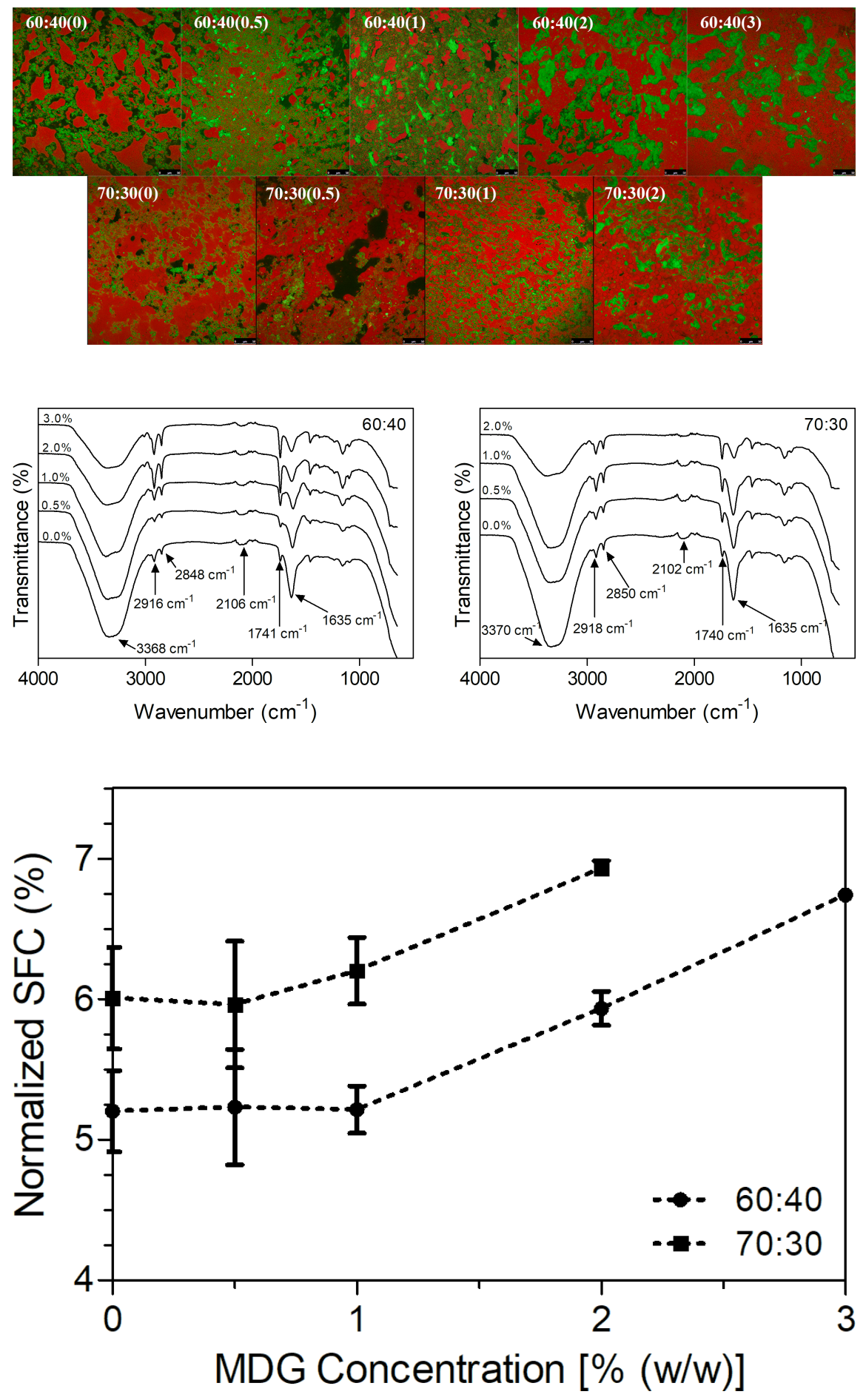

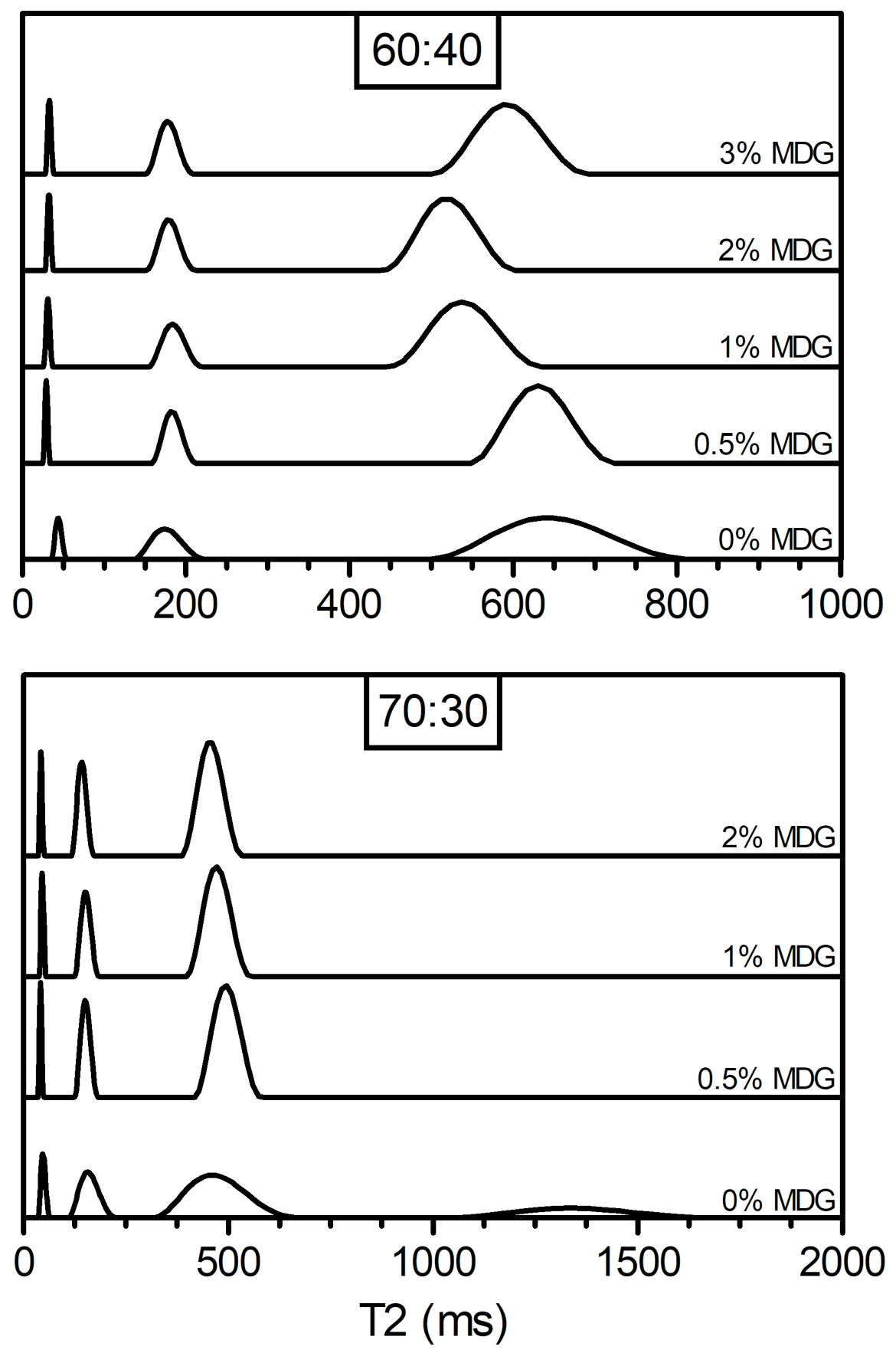


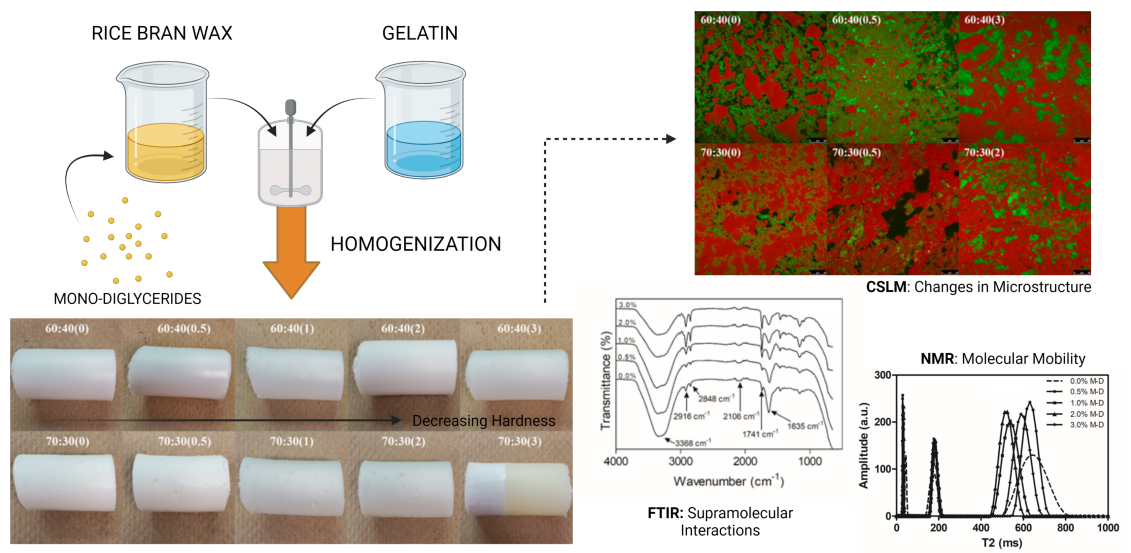

